\title{
Specificity of Sensory and Motor Neurons Associated with BL40 and GB30 in the Rat: A Dual Fluorescent Labeling Study
}

\author{
Jingjing Cui, ${ }^{1}$ Lijuan $\mathrm{Ha}^{2}{ }^{2}$ Xinlong Zhu, ${ }^{1}$ Fuchun Wang, ${ }^{2}$ \\ Xianghong Jing, ${ }^{1}$ and Wanzhu Bai ${ }^{1}$ \\ ${ }^{1}$ Institute of Acupuncture and Moxibustion, China Academy of Chinese Medical Sciences, Beijing 100700, China \\ ${ }^{2}$ Changchun University of Chinese Medicine, Changchun 130117, China
}

Correspondence should be addressed to Wanzhu Bai; wanzhubaisy@hotmail.com

Received 5 June 2013; Accepted 23 July 2013

Academic Editor: Yong-Qing Yang

Copyright (C) 2013 Jingjing Cui et al. This is an open access article distributed under the Creative Commons Attribution License, which permits unrestricted use, distribution, and reproduction in any medium, provided the original work is properly cited.

\begin{abstract}
The purpose of this study is to investigate the specific innervations on "Weizhong" (BL40) and "Huantiao" (GB30) by using a dual neural tracing technique. After Alexa Fluor 488 and 594 conjugates of cholera toxin subunit B (AF488/594-CTB) were, respectively, injected into BL40 and GB30 in the same rat, the labeled sensory and motor neurons were examined in the rat's dorsal root ganglia (DRGs) and spinal cord at thoracic (T) and lumbar (L) segments with a laser scanning confocal microscope. In the cases of BL40 injection, AF488-CTB labeled sensory and motor neurons were located in $\mathrm{L}_{2-6}$ DRGs and on the mediolateral part of spinal ventral horn from $\mathrm{L}_{3}$ to $\mathrm{L}_{5}$ segments, respectively. By contrast, in the cases of GB30 injection, AF594-CTB labeled sensory and motor neurons were distributed in $\mathrm{T}_{13}-\mathrm{L}_{6}$ DRGs and on the anterolateral part of spinal ventral horn from $\mathrm{L}_{1}$ to $\mathrm{L}_{5}$ segments, respectively. These results indicate that the sensory and motor neurons associated with BL40 and GB30 are located in different spinal segments and regions in the nervous system, providing the neuroanatomical evidence to serve the specificity of acupoints.
\end{abstract}

\section{Introduction}

Both "Weizhong" (BL40) and "Huantiao" (GB30) are commonly used for acupuncture treatment on lumbar and lower limb disorders [1-3], which could be traced back to "A Verse on the Twelve Heaven-Star Points" compiled by Ma Danyang in Song dynasty, showing its important historical position in acupuncture treatment. Although BL40 and GB30 play active roles in treating common ailments, such as sciatica, lumbar intervertebral disc herniation, and muscle strain [13 ], the underlying mechanism for their effectiveness remains unclear. Recent studies suggest that the effect of acupuncture is highly correlated with the activation of the nervous system [4-8]. Thus, to understand the neural properties of different acupoints may play a pivotal role in exploring the mechanism of acupuncture. In line with this kind of studies, how to apply an effective approach for further investigating the specific innervations on different acupoints is an important task to know the specificity of acupoints.

Since neural tracing technique was introduced into the acupuncture research, it has opened a new field to understand the neural properties of acupoints at the cellular level, especially the sensory and motor innervation on acupoints [911]. As a new generation of sensitive tracer $[12,13]$, Alexa Fluor 488 and 594 conjugates of cholera toxin subunit B (AF488/594-CTB) have been proven to be a potential approach in morphological study on the neural properties of acupoints $[14,15]$. In order to reveal the characteristics of the neurons related to different acupoints, in this study, BL40 and GB30 were, respectively, labeled AF488/594-CTB in rats. By using this neural tracing technique, the distribution of the sensory and motor neurons associated with BL40 and GB30 could be revealed, which should benefit the understanding of the specificity of acupoints from the perspective of neuroanatomy.

\section{Material and Methods}

2.1. Subjects. Four adult male Sprague Dawley rats (6-7 weeks old, weight $225 \pm 25 \mathrm{~g}$ ) at clean level were used in the present study. Experimental animals were provided by the Institute of Laboratory Animal Sciences, Chinese Academy of Medical 


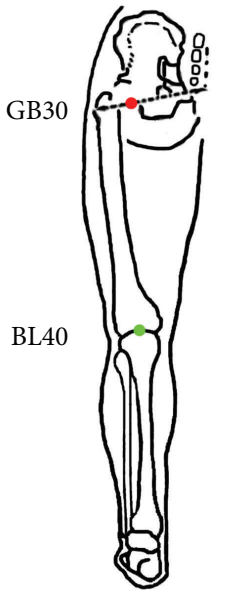

(a)

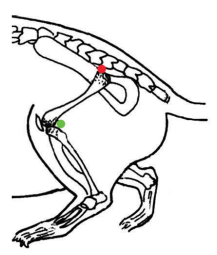

(b)
FIGURE 1: Illustration of the acupoints of Huantiao (GB30, red) and Weizhong (BL40, green) in human (a) and rat (b).

Sciences. The license number is SCKX (JUN) 2007-004. All animals were housed in a $12 \mathrm{~h}$ light/dark cycle with controlled temperature and humidity and allowed free access to food and water. The handling and care of experimental animals conformed to the regulations provided by the National Institutes of Health Guide for the Care and Use of Laboratory Animals [16].

\subsection{Research Protocol}

2.2.1. Microinjection of AF488/594-CTB. Injection was performed on both BL40 and GB30 in the same rat. Based on the classification of traditional Chinese medicine, BL40 belongs to the bladder meridian of foot Taiyang and GB30 is located on the gallbladder meridian of foot Shaoyang. The AF488/594-CTB (Invitrogen-Molecular Probes, Eugene, OR, USA) were used as tracers to, respectively, determine the distribution of neurons related to BL40 and GB30. According to the principle of comparative anatomy, BL40 is located at the midpoint of the popliteal crease, and GB30 is situated at the junction of the lateral $1 / 3$ and medial $2 / 3$ of the line connecting the prominence of greater trochanter of femur with the sacral hiatus on the rat, corresponding to BL40 and GB30 on the human body (Figure 1). Under anesthesia with isoflurane (Litian, Jiupai Pharmaceutical Co. Ltd, Hebei, China) controlled by small animal anesthesia machine (VMR, Matrx, Midmark, USA), $8 \mu$ L of $0.1 \%$ AF488/594-CTB were, respectively, injected into BL40 and GB30 on the left side with $10 \mu \mathrm{L}$ Hamilton syringe. The depth of injection was 4-5 mm. In order to prevent leakage of solution, the needle was kept for $1 \mathrm{~min}$ after injection and then slowly pulled out. When the rats awoke from anesthesia, they were put back in their cages.

2.2.2. Perfusion. Three days $(72 \mathrm{~h})$ after injection, the rats were deeply anaesthetized by ether and transcardially perfused with $150 \mathrm{~mL}$ of $0.9 \%$ saline immediately followed by
$300 \mathrm{~mL}$ of $4 \%$ paraformaldehyde in $0.1 \mathrm{M}$ phosphate buffered solution ( $\mathrm{PB}, \mathrm{pH}$ 7.4). After perfusion, dorsal root ganglia (DRGs) and spinal cord from thoracic (T) 10 to lumbar (L) 6 segments were dissected out and put into the same fixative solution about $2-4 \mathrm{~h}$, then changed into $25 \%$ sucrose $\mathrm{PB}(0.1 \mathrm{M}, \mathrm{pH} 7.4)$ at $4^{\circ} \mathrm{C}$, and allowed to sink. The tissue on injection site was also dissected out for observing the local diffusion of tracers. The level of spinal segments was determined cytoarchitecturally, referred as to The Rat Brain in Stereotaxic Coordinates [17].

2.2.3. Section. Serial sagittal sections of DRGs and transverse sections of spinal cord were cut at a thickness of $40 \mu \mathrm{m}$ on a freezing microtome (Thermo, Microm International $\mathrm{GmbH}$, Germany). All sections were collected in order in a six-hole Petri dish with in $0.1 \mathrm{M} \mathrm{PB}(\mathrm{pH} 7.4)$ and then stored in the refrigerator at $4^{\circ} \mathrm{C}$.

Before observation, the sections were mounted on the microscope slides and coverslipped with 50\% glycerin to improve visualization of labeling.

2.2.4. Observation. The tissue samples were observed and recorded with a laser scanning confocal microscope (FV1000, Olympus Co., Tokyo, Japan). Digital images were then processed with Adobe Photoshop CS2 (Adobe Systems, San Jose, CA, USA).

2.2.5. Statistical Analysis. Data was expressed as mean \pm standard deviation and processed with the statistical software SPSS 16.0.

\section{Results}

The labeled sensory and motor neurons with AF488/594CTB were located ipsilaterally on the injection side, in which AF488-CTB labeling was demonstrated in fluorescent green, and AF594-CTB was shown in fluorescent red (Figure 2). The neurons associated with BL40 and GB30 were distributed separately on the thoracic and lumbar DRGs and spinal cord. No neural labeling was observed above the level of spinal cord. The segmental and regional distribution of the sensory and motor neurons associated with BL40 and GB30 was summarized in Figure 3.

3.1. Sensory Innervation. In the cases of BL40 injection, the AF488-CTB labeled sensory neurons were detected in $\mathrm{L}_{2}-\mathrm{L}_{6}$ DRGs with high concentration in $\mathrm{L}_{5}$ DRGs (Figures $2(\mathrm{~A})$ and 3). By contrast, in the cases of GB30 injection, the AF594CTB labeled sensory neurons were detected in the $\mathrm{T}_{13}-\mathrm{L}_{6}$ DRGs with high concentration in $\mathrm{L}_{4}$ DRGs (Figures 2(A1) and 3 ). No double labeled sensory neurons were detected (Figure 2(A2)). In the four rats, a total of 538 AF488-CTB labeled sensory neurons (BL40) and 866 AF594-CTB labeled sensory neurons (GB30) were counted in the DRGs and arranged in order at different segments (Figure 4).

According to the size of soma diameter, the labeled sensory neurons were assigned to three classes: the large one (soma diameter $>50 \mu \mathrm{m}$ ), the medium one (soma diameter between $30 \mu \mathrm{m}$ and $50 \mu \mathrm{m}$ ), or the small one 

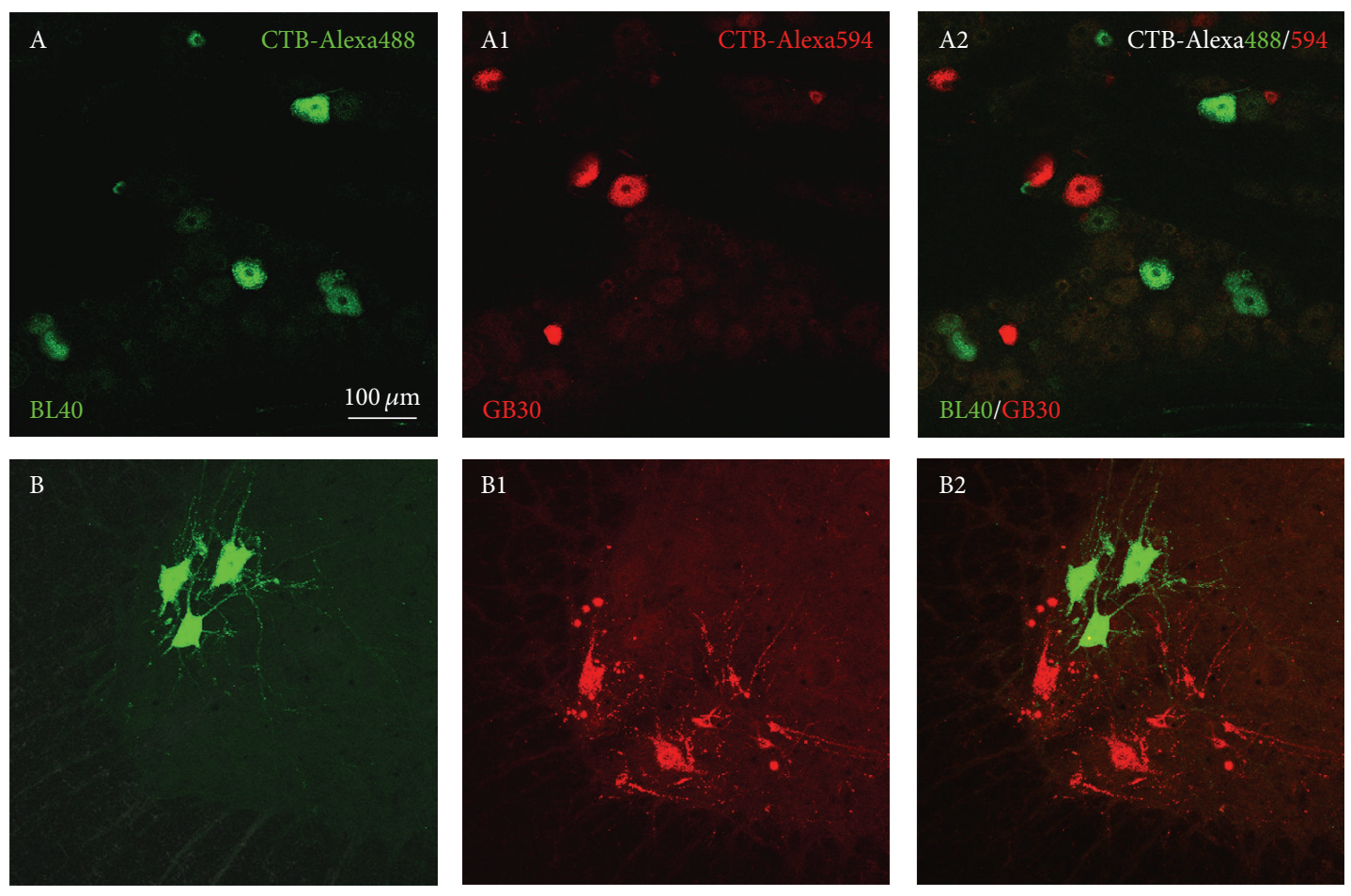

FIGURE 2: Representative sections from lumbar 4 dorsal root ganglion (DRG) and spinal cord showing the distribution of the labeled sensory neurons in DRG (A-A2) and motor neurons in spinal ventral horn (B-B2) after respective injection of AF488-CTB and AF594-CTB into BL40 and GB30 in the same rat. A, B: AF488-CTB labeled sensory (A) and motor (B) neurons related to BL40; A1, B1: AF594-CTB labeled sensory (A1) and motor (B1) neurons related to GB30; A2, B2: merged photo from A and A1(A2), B and B1(B2). Scale bar for all photos shown in A.

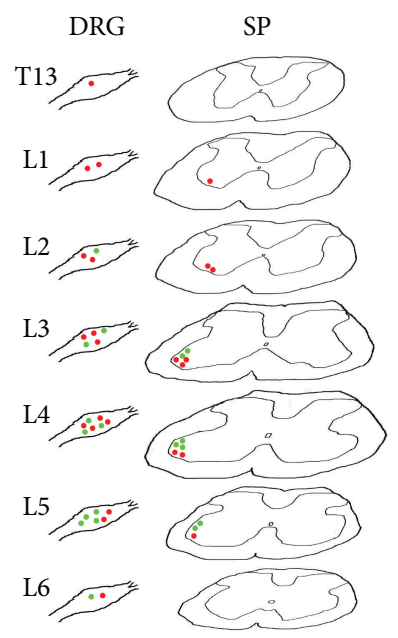

FIGURE 3: A series of line drawings through seven levels of dorsal root ganglia (DRGs) and approximately equal levels of spinal cord (SP) from thoracic (T) 13 to lumbar (L) 6 spinal segments showing the segmental and regional distribution of the sensory and motor neurons associated with BL40 (green) and GB30 (red), respectively.

(soma diameter $<30 \mu \mathrm{m}$ ) [18]. In both BL40 and GB30 injections, the small- and medium-sized sensory neurons each represented over $40 \%$ of the labeled neurons (Table 1 ).

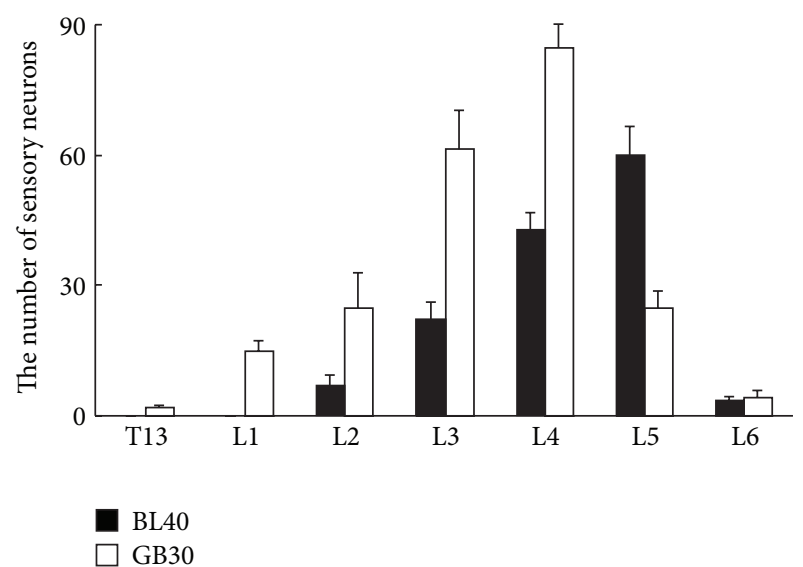

FIGURE 4: The average number of the AF488-CTB and AF594-CTB labeled sensory neurons in the DRGs ranging from thoracic (T) 13 to lumbar (L) 6 segments (mean \pm standard deviation, $n=4$ ), in which the sensory neurons associated with BL40 were distributed from L2 to L6 segments and those of GB30 distributed from T13 to L6.

3.2. Motor Innervation. In the cases of BL40 injection, the AF488-CTB labeled motor neurons were distributed on the mediolateral part of spinal ventral horn from $\mathrm{L}_{3}$ to $\mathrm{L}_{5}$ segments and concentrated at $\mathrm{L}_{4}$ segment (Figures $2(\mathrm{~B})$ and 3 ). By contrast, in the cases of GB30 injection, the AF594-CTB 
TABLE 1: Numbers and proportions of large-, medium-, and smallsized sensory neurons associated with BL40 and GB30 in the DRGs (number (\%)).

\begin{tabular}{lcc}
\hline Sensory neurons in the DRGs & BL40 & GB30 \\
\hline Large-sized neuron & $58(10.78 \%)$ & $149(17.21 \%)$ \\
Medium-sized neuron & $222(41.26 \%)$ & $357(41.22 \%)$ \\
Small-sized neuron & $258(47.96 \%)$ & $360(41.57 \%)$ \\
\hline
\end{tabular}

labeled motor neurons were distributed on the anterolateral part of spinal ventral horn from $\mathrm{L}_{1}$ to $\mathrm{L}_{5}$ segments with high concentration at $\mathrm{L}_{3}$ segment (Figures 2(B1) and 3). No double labeled motor neurons were observed (Figure 2(B2)). Because there is no distinct boundary among the different spinal segments, the figures were shown at approximate segment of spinal cord, and the labeled motor neurons were not counted separately.

According to the size of soma diameter, the labeled motor neurons were divided into two classes: the large one (soma diameter $>25 \mu \mathrm{m}$ ) or the small one (soma diameter $\leq 25 \mu \mathrm{m}$ ), belonging to $\alpha$ and $\gamma$ motor neurons, respectively [19]. The approximate 60 transverse sections from spinal cord were counted on every rat, resulting in a total of 136 AF488CTB labeled motor neurons (BL40) and 174 AF594-CTB labeled motor neurons (GB30). The large- and small-sized motor neurons, respectively, represented 98.53\% (134/136) and $1.47 \%(2 / 136)$ in the cases of BL40 injection and $92.53 \%$ $(161 / 174)$ and $7.47 \%(13 / 174)$ in the cases of GB30 injection.

In addition, around the injection site, AF488-CTB and AF594-CTB were diffused in the muscles and subcutaneous tissues, but the scope of local diffusion was not over $1 \mathrm{~mm}$ from the center of injection site.

\section{Discussion}

By using a dual fluorescent labeling technique with AF488/ 594-CTB, we successfully revealed that the sensory and motor neurons associated with BL40 and GB30 were distributed separately in DRGs and spinal cord in rats, in which the segmental and regional arranged neurons closely correspond with individual acupoint, providing the neuroanatomical evidence to serve the specificity of acupoints.

4.1. Technical Considerations. Recently, AF488/594-CTB are increasingly used for tracing the neural pathway in neuroscience research $[12,13]$. Our recent studies also suggested that the technique of AF488/594-CTB is a proper choice to investigate the neural properties of different acupoints [14, 15]. Compared with tracers previously used in this field, such as propidium iodide (PI) and bisbenzimide (Bb) [20-22], the main advantages of AF488/594-CTB are their sensitivity and fluorescence persistence. Because the spectral range of specific fluorescent materials carried by AF488/594-CTB is concentrated and corresponds with the stimulating lights of a laser scanning confocal microscope, its neural labeling is more easily observed and identified without disturbance of nonspecific fluorescence labeling as observed with that of PI and $\mathrm{Bb}$ labeling [20-22]. Although it does not need complex staining processes to detect neural labeling of AF488/594CTB like that of Horseradish Peroxidase (HRP) [11], it should be noted that AF488/594-CTB are only limited to the application of retrograde labeling the sensory and motor neurons in the tracing study and cannot be used for labeling transganglionic axonal terminals like that of HRP. Nevertheless, the present results provided sufficient evidence to propose that AF488/594-CTB are a suitable couple for dual fluorescent labeling in morphological research of acupuncture.

4.2. Distribution of Sensory and Motor Neurons. Previous topographic studies have shown that BL40 and GB30 are highly correlated with the tibial nerve and sciatic nerve, respectively, at the level of gross anatomy $[23,24]$. However, at the cellular level, we still do not know the distribution of the sensory and motor neurons associated with both acupoints in the nervous system. In this study, we demonstrated that neurons related to BL40 and GB30 were distributed in a definite segmental and regional pattern, in which the sensory and motor neurons associated with GB30 distributed more extensively and higher than those of BL40 about one or two segments in DRGs and spinal ventral horn (Figure 3). Although with a part of segmental overlap, there were no dual labeled neurons in the present study. Thus it could be concluded that BL40 and GB30 received innervations from different sensory and motor neurons. In addition, the motor neurons related to BL40 and GB30 were distributed in the mediolateral and anterolateral regions of spinal ventral horn, respectively. This regional arrangement also supports the idea that the innervations on different acupoints originate from different neurons.

It has been shown that peripheral areas on the body correlate orderly with the nervous system, which was named somatotopic organization [25-27]. Without exception, as a point of peripheral areas, every acupoint has its own corresponding neurons in the nervous system. Considering the distances from BL40 and GB30 to the truck of body, it is clear that the closer to the body trunk the acupoint is, the higher the neurons associated with the corresponding acupoint situate in the spinal segment. Similar results were also demonstrated in our previous observations on the acupoints of "Taixi" (KI3), "Chengshan" (BL 57), "Jinggu" (BL64), and "Dazhong" (KI4) $[11,14,15]$. Through these neural tracing studies on the different acupoints, we can speculate the innervations on acupoints according to their locations on the proximal, mid, and distal parts of hindlimb. Therefore, from the perspective of neuroanatomy, this study increases our understanding of the regular connections between the different acupoints and the nervous system.

4.3. The Subtype of Sensory and Motor Neurons. Large-, medium-, and small-sized sensory neurons were simultaneously labeled in both cases of BL40 and GB30 injections, which roughly correspond to $\mathrm{A} \alpha / \mathrm{A} \beta-, \mathrm{A} \delta$-, and $\mathrm{C}$-fibers, respectively $[18,28]$. These three types of sensory neurons may play different roles in the processing of signals transmission due to their electrophysiological properties [29-31]. It was suggested that $\mathrm{A} \alpha / \mathrm{A} \beta$-type fibers transmit messages of tactile sensation and proprioception, while $\mathrm{A} \delta$ - and C-type fibers 
relay messages of nociception and thermal sensation [29-31]. Since different kinds of sensory neurons directly innervate the acupoints, it may be an important implication for further considering roles of these subtype neurons in the process of acupuncture stimulation. Besides the different kinds of sensory neurons, both $\alpha$ and $\gamma$ motor neurons also participate in innervation on the BL40 and GB30. It should be another important consideration on the neural properties of different acupoints. Although we only provided the neuroanatomical evidence of BL40 and GB30 in this study, these results have served as a source of inspiration for investigating the specificity of acupoints at the cellular level.

4.4. The Significance for Clinical Practice. The relative specificity of acupoints is an important issue in the clinical treatment. To understand the neural properties of BL40 or GB30 should be of benefit for us to select proper one for acupuncture treatment according to the patient's symptoms. Given that the neurons associated with BL40 and GB30 concentrate on different spinal segments, we suggest that when the lumbar disorder occurred on $\mathrm{L}_{4}, \mathrm{~GB} 30$ was recommended as main acupoint, while if it occurred on $\mathrm{L}_{5}$, BL40 was recommended. We can also simultaneously select both GB30 and BL40 to treat the ailment involving multiple spinal segments. Although this implication was supported by the perspective of neural pathway from the different acupoints to the nervous system, it remains to be verified in clinical practice.

\section{Conclusion}

In summary, we successfully demonstrated the specificity of distribution and subtype of the sensory and motor neurons associated with BL40 and GB30 in the rat by using a dual fluorescent labeling technique with AF488/594-CTB. These results suggest that regular connections between the different acupoints and the nervous system should be an important consideration during the acupuncture treatment.

\section{Acknowledgments}

This study was funded by the National Natural Science Foundation of China (Project no. 81072759) and the National Basic Research Program of China (973 Program, no. 2010CB530507; no. 2011CB505201) and the Self-Selected Research Program from China Academy of Chinese Medical Sciences (no. 2009Z02024).

\section{References}

[1] R. Q. Gao, "168 cases of acupuncturing Huantiao and Weizhong on sciatica," Hebei Journal of Traditional Chinese Medicine, vol. 24, no. 1, p. 41, 2002.

[2] R. Zou, Y. Xu, and H. Zhang, "Evaluation on analgesic effect of electroacupuncture combined with acupoint-injection in treating lumbar intervertebral disc herniation," China Journal of Orthopaedics and Traumatology, vol. 22, no. 10, pp. 759-761, 2009.

[3] M. Hu and R. Zhang, "Clinical observation on warmingpromotion acupuncture for lumbar muscle strain," Chinese Acupuncture \& Moxibustion, vol. 31, no. 7, pp. 622-624, 2011.
[4] Z. Zhao, "Neural mechanism underlying acupuncture analgesia," Progress in Neurobiology, vol. 85, no. 4, pp. 355-375, 2008.

[5] C. Y. Chen, R. S. Chern, M. H. Liao, Y. H. Chang, J. Y. C. Hsu, and C. H. Chien, "The possible neuronal mechanism of acupuncture: morphological evidence of the neuronal connection between groin A-shi point and uterus," Evidence-Based Complementary and Alternative Medicine, vol. 2013, Article ID 429186, 13 pages, 2013.

[6] S. X. Ma, "Neurobiology of acupuncture: toward CAM," Evidence-Based Complementary and Alternative Medicine, vol. 1, no. 1, pp. 41-47, 2004.

[7] S. Uchida and H. Hotta, "Acupuncture affects regional blood flow in various organs," Evidence-Based Complementary and Alternative Medicine, vol. 5, no. 2, pp. 145-151, 2008.

[8] Z. Zhang, X. Wang, and G. M. McAlonan, "Neural acupuncture unit: a new concept for interpreting effects and mechanisms of acupuncture," Evidence-Based Complementary and Alternative Medicine, vol. 2012, Article ID 429412, 23 pages, 2012.

[9] Z. L. Tao, Z. P. Zhang, L. P. Wang, C. H. Li, and B. Z. Wen, "The segmentation of the afferent neurons of the area of 'Zusanli," Acupuncture Research, vol. 8, no. 2, pp. 136-140, 1983.

[10] S. Y. Xi and Z. L. Tao, "Afferent fibers of the points of three Yang channels of the foot in the lower limbs projected to the spinal cord and medulla oblongata," Acupuncture Research, no. 2, pp. 130-134, 1983.

[11] J. Jiang, W. Bai, L. Zhang, X. Jing, and Z. Jin, "Segmental and regional innervation of acupoint "Taixi" (KI 3) area in the rat: a horseradish peroxidase method study," Acupuncture Research, vol. 35, no. 1, pp. 27-36, 2010.

[12] W. L. Conte, H. Kamishina, and R. L. Reep, "Multiple neuroanatomical tract-tracing using fluorescent Alexa Fluor conjugates of cholera toxin subunit B in rats," Nature Protocols, vol. 4, no. 8, pp. 1157-1166, 2009.

[13] J. A. Christianson, R. Liang, E. E. Ustinova, B. M. Davis, M. O. Fraser, and M. A. Pezzone, "Convergence of bladder and colon sensory innervation occurs at the primary afferent level," Pain, vol. 128, no. 3, pp. 235-243, 2007.

[14] X. Zhu, W. Bai, F. Wu, J. Jiang, and X. Jing, "Neuroanatomical characteristics of acupoint "Chengshan" (BL 57) in the rat: a cholera toxin subunit B conjugated with Alexa Fluor 488 method study," Acupuncture Research, vol. 35, no. 6, pp. 433-437, 2010.

[15] J. Cui, X. Zhu, C. Ji, X. Jing, and W. Bai, "Neuroanatomical basis of clinical joint application of "Jinggu" (BL 64, a source-acupoint) and "Dazhong" (KI 4, a Luo-acupoint) in the rat: a double-labeling study of cholera toxin subunit B conjugated with Alexa Fluor 488 and 594," Acupuncture Research, vol. 36, no. 4, pp. 262-267, 2011.

[16] National Research Council, Guide for the Care and Use of Laboratory Animals, National Academic Press, Washington, DC, USA, 1996.

[17] G. Paxinos and C. Watson, The Rat Brain in Stereotaxic Coordinates, Academic Press, San Diego, Calif, USA, 1998.

[18] J. M. Caffrey, D. L. Eng, J. A. Black, S. G. Waxman, and J. D. Kocsis, "Three types of sodium channels in adult rat dorsal root ganglion neurons," Brain Research, vol. 592, no. 1-2, pp. 283-297, 1992.

[19] R. R. Roy, A. Matsumoto, H. Zhong, A. Ishihara, and V. R. Edgerton, "Rat $\alpha$ - and $\gamma$-motoneuron soma size and succinate dehydrogenase activity are independent of neuromuscular activity level," Muscle and Nerve, vol. 36, no. 2, pp. 234-241, 2007. 
[20] C. G. Tong, S. Z. Gu, H. Q. Yi, C. Q. Guo, X. R. Xiang, and B. $\mathrm{Xu}$, "Research on the specific pathways connecting stomach and its Back-Shu and Front-Mu points by using fluorescent double labeling method," Shanghai Journal of Acupuncture and Moxibustion, vol. 22, no. 5, pp. 16-19, 2003.

[21] J. L. Zhang, S. P. Chen, and J. L. Liu, "Research on the connection pathway between "Tianquan" (PC 2) of the "Pericardium Meridian" and the heart in the cat," Acupuncture Research, vol. 27, no. 2, pp. 124-129, 2002.

[22] L. Zhang, J. Jiang, Z. Jin, and J. Liu, "Afferent nerve connection among "Hegu" (LI4), "Neiguan" (PC6), "Futu" (LI18) and thyroid gland region: fluorescent double labelling method," Acupuncture Research, vol. 35, no. 6, pp. 438-442, 2010.

[23] H. R. Cui, X. F. Lou, K. Y. Dai, S. W. Hu, S. H. Jiang, and X. D. Yang, "Topographic research of Weizhong Acupoint (BL40) and its clinical significance," Journal of Wenzhou Medical College, vol. 37, no. 3, pp. 224-226, 2007.

[24] W. X. Qiu, S. X. Duan, L. P. Hou et al., "Anatomical research of Huantiao Acupoint (GB30) and the sciatic nerve," Journal of Clinical Acupuncture and Moxibustion, vol. 15, no. 4, pp. 57-58, 1999.

[25] C. Rivero-Melian, "Organization of hindlimb nerve projections to the rat spinal cord: a choleragenoid horseradish peroxidase study," The Journal of Comparative Neurology, vol. 364, no. 4, pp. 651-663, 1996.

[26] C. Molander and G. Grant, "Laminar distribution and somatotopic organization of primary afferent fibers from hindlimb nerves in the dorsal horn. A study by transganglionic transport of horseradish peroxidase in the rat," Neuroscience, vol. 19, no. 1, pp. 297-312, 1986.

[27] Y. Takahashi, T. Chiba, M. Kurokawa, and Y. Aoki, "Dermatomes and the central organization of dermatomes and body surface regions in the spinal cord dorsal horn in rats," Journal of Comparative Neurology, vol. 462, no. 1, pp. 29-41, 2003.

[28] A. A. Harper and S. N. Lawson, "Conduction velocity is related to morphological cell type in rat dorsal root ganglion neurones," Journal of Physiology, vol. 359, pp. 31-46, 1985.

[29] T. L. Yaksh and D. L. Hammond, "Peripheral and central substrates involved in the Rostrad transmission of nociceptive information," Pain, vol. 13, no. 1, pp. 1-85, 1982.

[30] A. A. Harper and S. N. Lawson, "Electrical properties of rat dorsal root ganglion neurones with different peripheral nerve conduction velocities," Journal of Physiology, vol. 359, pp. 47-63, 1985.

[31] P. J. Waddell and S. N. Lawson, "Electrophsyiological properties of subpopulations of rat dorsal root ganglion neurons in vitro," Neuroscience, vol. 36, no. 3, pp. 811-822, 1990. 


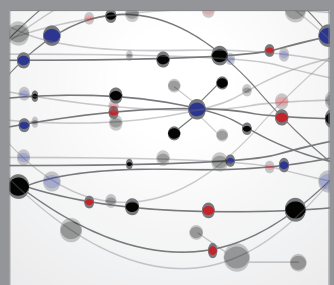

The Scientific World Journal
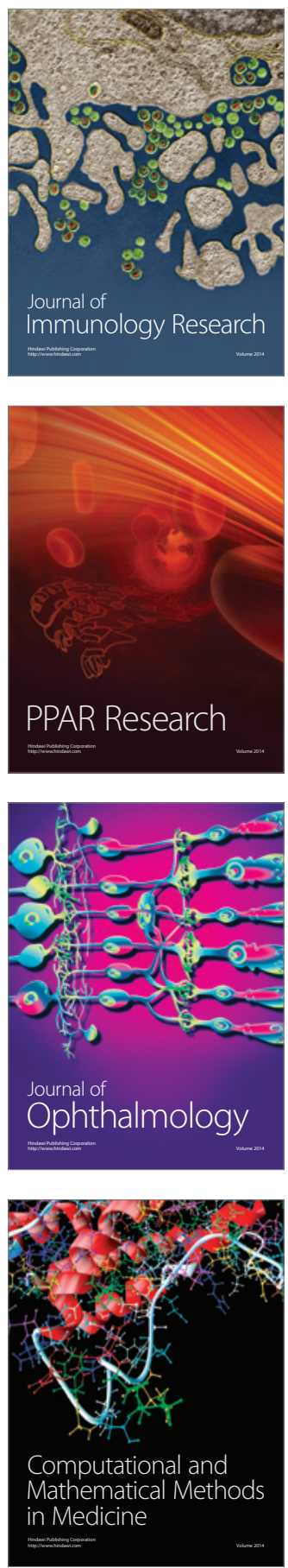

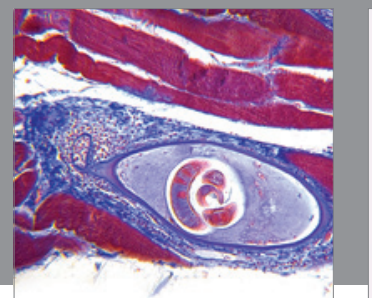

Gastroenterology

Research and Practice
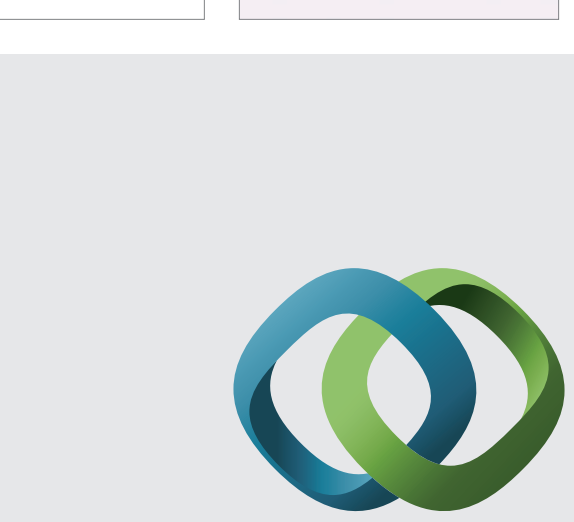

\section{Hindawi}

Submit your manuscripts at

http://www.hindawi.com
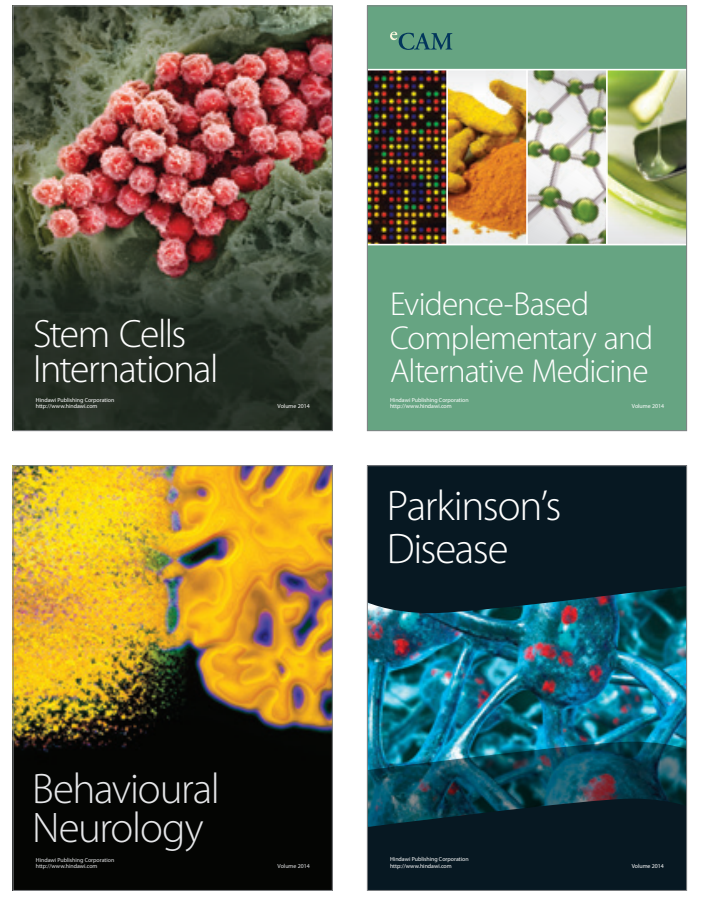
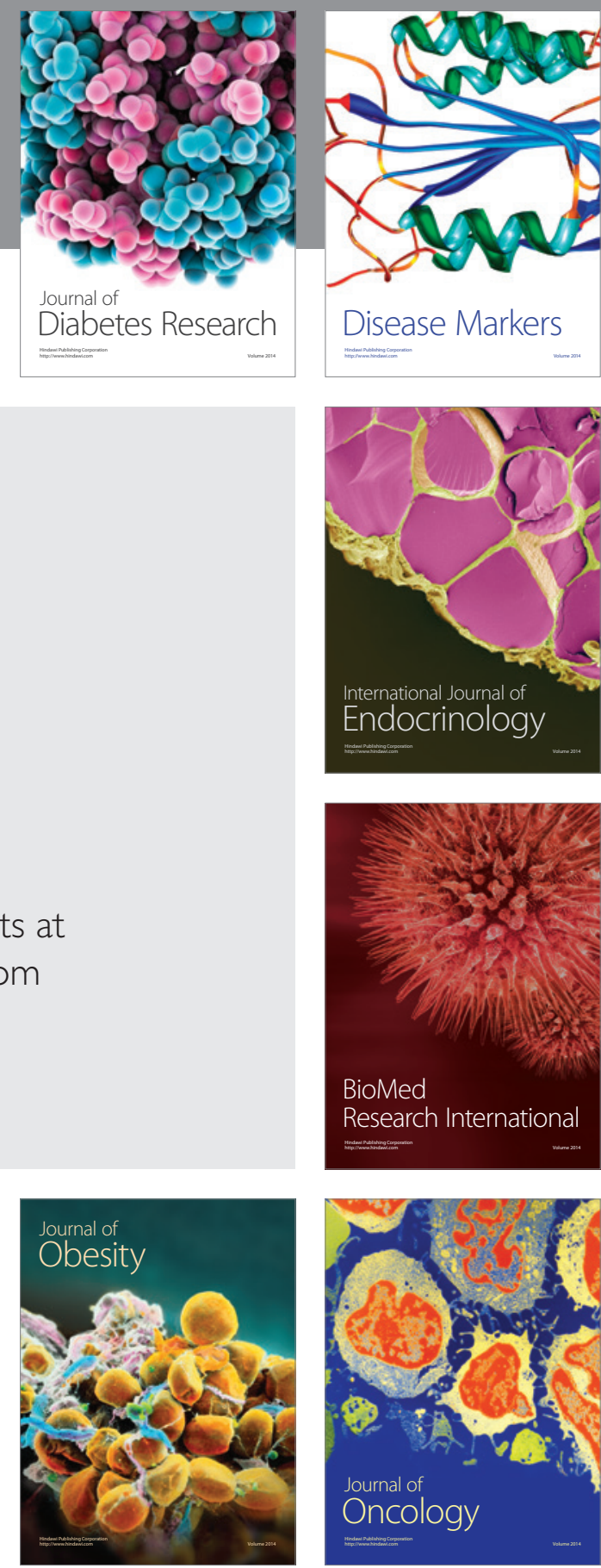

Disease Markers
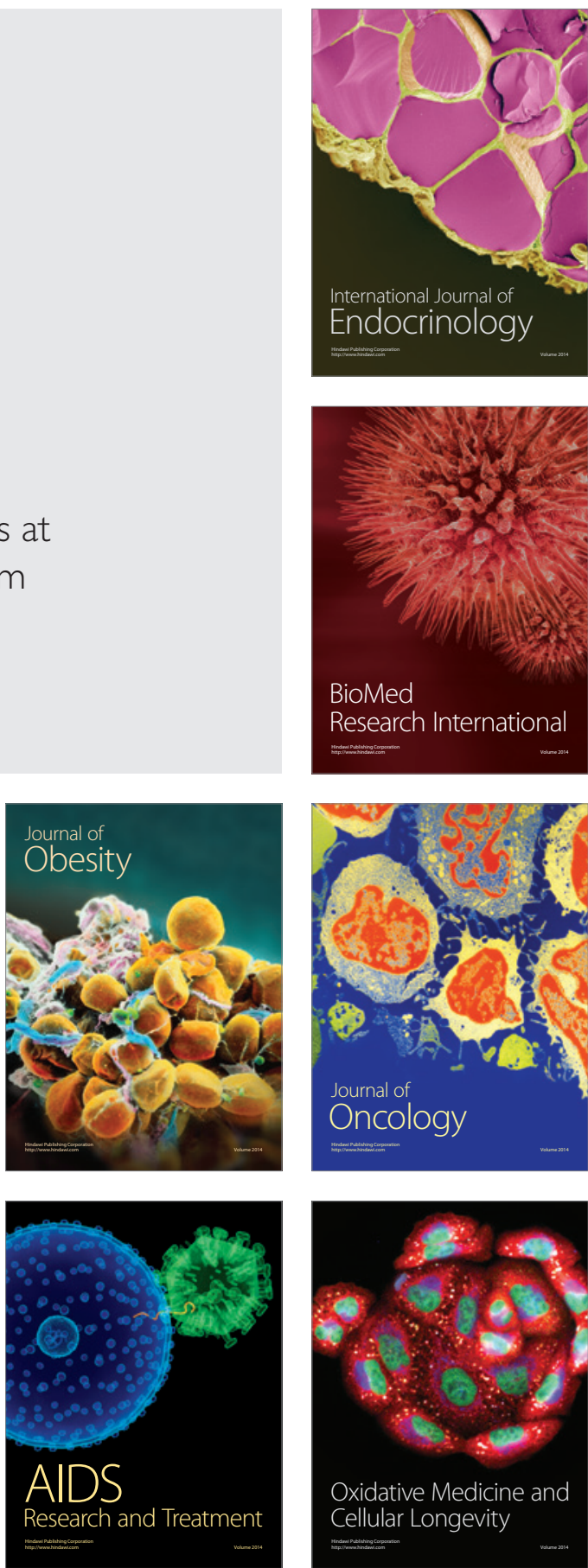